tion $^{3}$ or bronchoplasty procedures in patients with lung cancer after induction therapy ${ }^{2}$ for the same purpose.

\section{References}

1. Okada M, Tsubota N, Yoshimura M, Miyamoto Y, Matsuoka H, Satake S, et al. Extended sleeve lobectomy for lung cancer: the avoidance of pneumonectomy. J Thorac Cardiovasc Surg. 1999;118: 710-4.

2. Ohta M, Sawabata N, Maeda H, Matsuda H. Efficacy and safety of tracheobronchoplasty after induction therapy for locally advanced lung cancer. J Thorac Cardiovasc Surg. 2003;125:96-100.

3. Calhoon JH, Grover FL, Gibbons WJ, Bryan CL, Levine SM, Bailey SR, et al. Single lung transplantation: alternative indications and technique. J Thorac Cardiovasc Surg. 1991;101:816-25.

\title{
Simultaneous heart valve replacement and reconstruction of the radiation-damaged chest wall with a delayed vertical rectus abdominis myocutaneous flap
}

\author{
Thomas Strecker, MD, ${ }^{a}$ Richard Feyrer, MD, ${ }^{a}$ Raymund E. Horch, MD, ${ }^{b}$ Michael Weyand, MD, ${ }^{a}$ and Ulrich Kneser, MD, \\ Erlangen, Germany
}

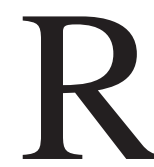
adiation ulcer of the chest wall after breast cancer treatment represents a severe physiologic and psychological problem. Patients with radiation ulcer who require cardiac surgery are at high risk for development of consecutive sternum osteomyelitis. In this article, we report a successful surgical aortic valve replacement and mitral valve reconstruction in combination with simultaneous reconstruction of the radiation-damaged chest wall using a delayed superiorly based vertical rectus abdominis myocutaneous flap in a 71-year-old patient. Cardiac surgery and plastic surgical reconstruction of the chest wall in a joint approach at the same time allow for safe and efficient treatment and may help to reduce the total number of operations and hospital stays.

\section{Clinical Summary}

A 71-year-old white woman with suspected endocarditis and symptoms of heart failure was referred to the cardiology department of our hospital. She noted progressive dyspnea, arterial hypertension, fatigue, and exhaustion. Twenty years ago, a right-sided radical mastectomy, followed by irradiation of the chest wall, was performed for treatment of breast cancer. Five

\footnotetext{
From the Center of Cardiac Surgery a and the Department of Plastic and Hand Surgery, ${ }^{\mathrm{b}}$ Friedrich-Alexander-University of Erlangen-Nuremberg, Erlangen, Germany,

Received for publication March 18, 2006; revisions received April 22, 2006; accepted for publication May 17, 2006.

Address for reprints: Thomas Strecker, MD, Center of Cardiac Surgery, Friedrich-Alexander-University of Erlangen-Nuremberg, Krankenhausstr 12, D-91054 Erlangen, Germany (E-mail: thomas.strecker@ herz.imed.unierlangen.de)

J Thorac Cardiovasc Surg 2006;132:980-2

$0022-5223 / \$ 32.00$

Copyright $\odot 2006$ by The American Association for Thoracic Surgery doi:10.1016/j.jtcvs.2006.05.063
}

years later, progressive skin fibrosis developed. Within the last 7 years before admission, the patient reported recurrent parasternal skin ulcerations with fistula.

At admission to the hospital, the laboratory tests showed a leukocytosis of $11.42 \times 10^{3} / \mu \mathrm{L}$ (normal range, $<10 \times 10^{3} / \mu \mathrm{L}$ ), and the other values were within the normal range. Physical examination revealed a $13 \times 9-\mathrm{cm}$ parasternal unstable scar with skin atrophy (Figure 1). The skin was attached to the rib cage because the pectoralis major muscle had been resected previously. On admission, there was no fistula visible. Transesophageal echocardiography revealed a severe aortic valve stenosis with maximum/mean pressure gradients of 74/43 $\mathrm{mm} \mathrm{Hg}$ and an aortic valve orifice of $0.84 \mathrm{~cm}^{2}$. Furthermore, there was a mediocre mitral valve insufficiency. There were no signs of endocarditis. The cardiac catheterization showed no significant stenoses of the coronary arteries and an ejection fraction of $44 \%$. Radiography and magnetic resonance imaging of the sternum disclosed osteolysis of the central third of the sternum either caused by osteomyelitis or aseptic osteoradionecrosis and fibrosis of the surrounding soft tissue without any evidence of mediastinitis.

Seven days before cardiac surgery, a right-sided vertical rectus abdominis myocutaneous (VRAM) flap (dimension, $10 \times 26 \mathrm{~cm}$ ) was delayed. The skin island was incised, and the inferior epigastric artery was ligated (Figure 1). Seven days later, the patient was again taken to the operating theater, where a median sternotomy was performed, and cardiopulmonary bypass was installed through aorto-right atrium cannulation. The calcified aortic valve was excised and replaced with a biologic prosthesis with a $23-\mathrm{mm}$ diameter. This mitral valve insufficiency was away with implantation of an annuloplastic ring with a 28-mm diameter. Intraoperatively, there were no signs of any myocarditis, endocarditis, or sternum osteomyelitis, and therefore the chest was closed with cerclage. The unstable parasternal and presternal scar was excised, and the delayed superiorly based VRAM flap was completely raised and transferred into the defect. The inferior part of the anterior rectus sheath was reconstructed with a polypropylene surgical mesh, and wounds were closed in layers at the recipient and donor sites. 


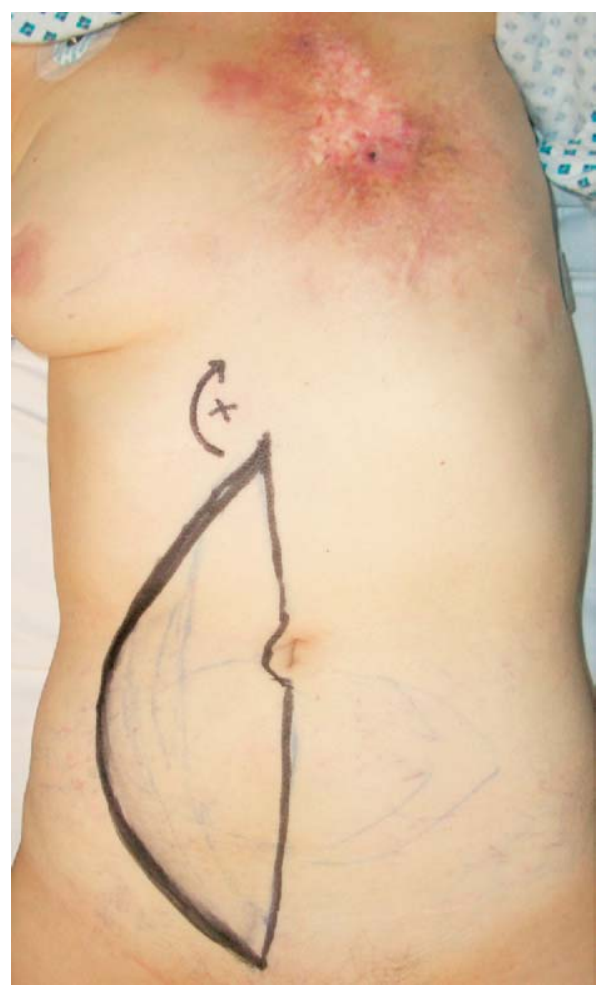

Figure 1. The unstable parasternal scar $(9 \times 13 \mathrm{~cm})$ after irradiation of the chest wall 20 years previously and the flap design before delay procedure. The dimensions of the flap are $26 \times 10 \mathrm{~cm}$. $X$, Prospective pivot point of the flap.

Postoperatively, the patient's cardiopulmonary status progressively deteriorated over the subsequent 24 hours. An intraaortic balloon pump was implanted over a period 5 days. Subsequently, it was possible to taper and withdraw inotropic support with stable hemodynamic parameters over the following days. The patient was extubated on day 2 after surgical intervention and transferred back to regular care on postoperative day 7. Histopathologic examination of the excised skin revealed skin fibrosis without evidence of Marjolin's ulcer. Before leaving the hospital, echocardiography showed a regular function of the implanted biologic aortic valve and the mitral annuloplastic ring without any regurgitation or paravalvular leakage and no relevant pericardial effusion. The flap and the donor site healed by primary intention, and the patient reported considerable relief of "tension" in the chest after excision of the unstable scar. Five months after surgical intervention, the patient was in good health. A repeated echocardiography confirmed the previous findings. Stabile coverage of the sternum was achieved, and there was no instability of the abdominal wall (Figure 2).

\section{Discussion}

Radiation ulcers are ulcerations of soft tissues and possibly underlying bone that result as a complication of previous radi-

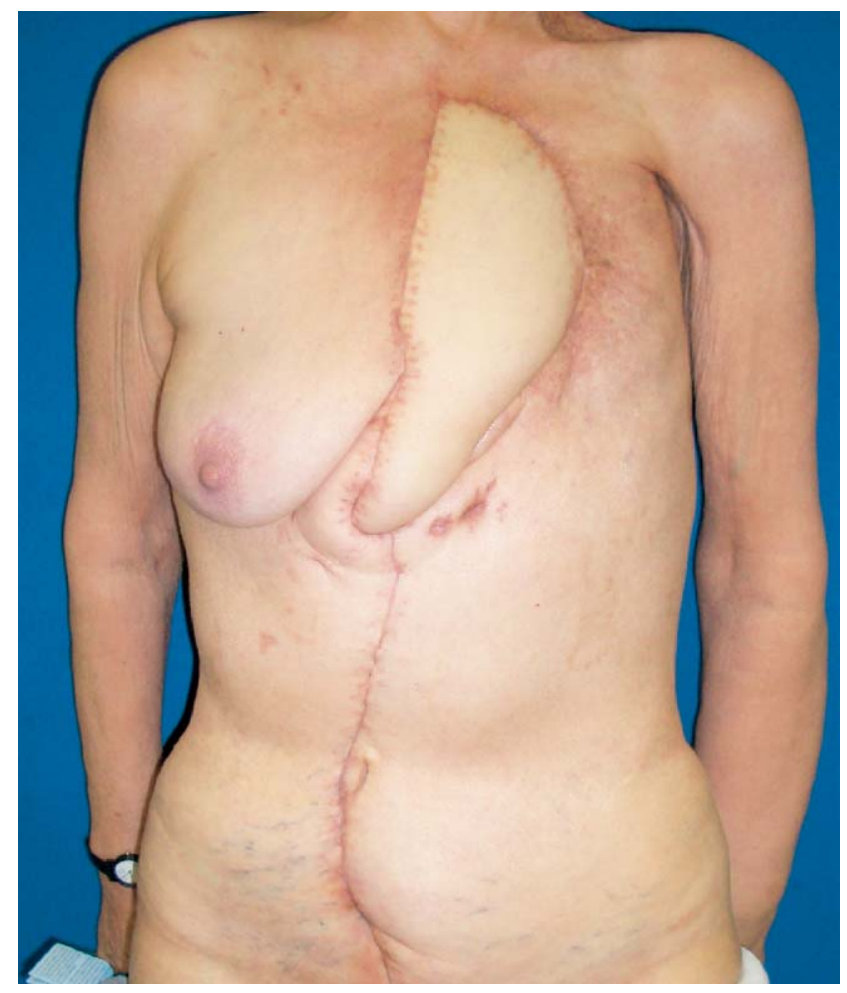

Figure 2. Five months after defect reconstruction. The entire flap is vital, without any sign of venous congestion. Wound healing was achieved by primary intention, and there was no clinical evidence of osteomyelitis or hernia.

ation exposure. ${ }^{1}$ In patients with an additional heart disease, the availability of cardiac surgery and plastic reconstruction at the same time make it possible to reduce time-consuming medical procedures. The VRAM flap is a well-established flap for reconstruction of sternotomy wounds and other chest wall defects. ${ }^{2}$ It is a valuable tool for treatment of sternum osteomyelitis after cardiac or thoracic surgery. Although the superiorly based VRAM flap is considered a safe flap with predictable outcome, the deep inferior epigastric system is the dominant pedicle of the rectus abdominis myocutaneous flap. ${ }^{3}$ Therefore extended VRAM flaps are at some risk for necrosis for the most caudal proportion of the skin island. The delay procedure efficiently increases the reliability of superiorly based transverse rectus abdominus myocutaneous flaps for breast reconstruction. Recently, the delay procedure has also been applied in one patient with radiation ulcer of the anterior chest wall. ${ }^{4}$ Fujiwara and colleagues ${ }^{4}$ demonstrated augmented blood supply of the VRAM flap after delay by means of angiography. In our hands the delayed, extended, superiorly based VRAM flap is a safe flap, even if the skin island is extended up to the symphysis pubis.

In conclusion, the presented interdisciplinary joint approach allows safe and efficient treatment of patients who are at high risk for postoperative complications, such as sternum osteomyelitis or wound dehiscence. 


\section{References}

1. Arnold PG, Pairolero PC. Reconstruction of the radiation-damaged chest wall. Surg Clin North Am. 1989;69:1081-9.

2. Coleman JJ 3rd, Bostwick J. Rectus abdominis muscle-musculocutaneous flap in chest-wall reconstruction. Surg Clin North Am. 1989;69:1007-27.
3. Moon HK, Taylor GI. The vascular anatomy of rectus abdominis musculocutaneous flaps based on the deep superior epigastric system. Plast Reconstr Surg. 1988;82:815-32.

4. Fujiwara M, Nakamura Y, Sano A, Nakayama E, Nagasawa M, Shindo T. Delayed vertical rectus abdominis myocutaneous flap for anterior chest wall reconstruction. Aesthetic Plast Surg. 2006;30:120-4.

\title{
Combined bronchial sleeve resection and repair of partial anomalous pulmonary venous return
}

\author{
Robert L. Smith II, MD, George L. Zorn III, MD, Benjamin B. Peeler, MD, and David R. Jones, MD, Charlottesville, Va
}

$\mathrm{P}$ ulmonary sleeve resection for benign and malignant tumors of the proximal lobar bronchi preserves pulmonary function and avoids performance of a pneumonectomy. Partial anomalous pulmonary venous return (PAPVR) in an adult is a rare finding that is usually asymptomatic. We report a case of an obstructing left lower lobe carcinoid tumor combined with a PAPVR of the left superior pulmonary vein that was successfully managed without the need for pneumonectomy.

\section{Clinical Summary}

A 45-year-old female nonsmoker presented with a several-month history of fatigue, night sweats, fever, and moderate dyspnea. Multiple antibiotic treatments for left lower lobe pneumonia had failed. A chest roentgenogram demonstrated a left hilar mass and lower lobe collapse. Bronchoscopy demonstrated a $2 \times 2-\mathrm{cm}$ mass nearly occluding the distal left main stem bronchus and originating from the left lower lobe bronchus. Bronchial brushings and washings were nondiagnostic, but the mass was highly suspicious for a carcinoid tumor.

Computed tomography demonstrated a normal-appearing left upper lobe, confirmed the left lower lobe collapse, and identified a presumed anomalous left superior pulmonary vein draining into the left brachiocephalic vein (Figure 1). Pulmonary function studies showed a forced expiratory volume in 1 second of $54 \%$ of predicted value, a forced vital capacity of $55 \%$ of predicted value, and a ratio of $76 \%$ with a normal diffusion capacity. Quantitative pulmonary perfusion nuclear imaging revealed $4 \%$ perfusion to the left lung, with $96 \%$ to the right lung. Echocardiography failed to demonstrate any intracardiac shunts.

From the University of Virginia School of Medicine, Charlottesville, Va. Received for publication May 8, 2006; accepted for publication May 17, 2006.

Address for reprints: David R. Jones, MD, Department of Surgery, General Thoracic Surgery, PO Box 800679, University of Virginia, Charlottesville, VA 22908-0679 (E-mail: djones@ virginia.edu).

J Thorac Cardiovasc Surg 2006;132:982-3

0022-5223/ $\$ 32.00$

Copyright $\odot 2006$ by The American Association for Thoracic Surgery doi:10.1016/j.jtcvs.2006.05.062

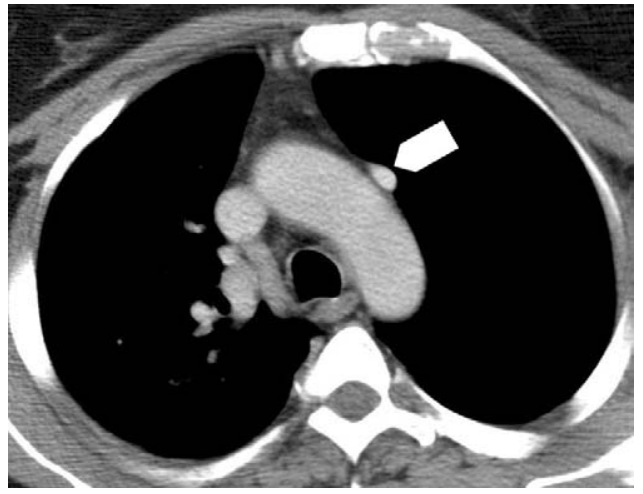

Figure 1. A preoperative computed tomogram identified an anomalous superior pulmonary vein (just lateral to the aortic arch and marked with an arrow) draining the left upper lobe and emptying into the left brachiocephalic vein.

Exploratory thoracotomy revealed a normal left upper lobe, a normal left inferior pulmonary vein, and a PAPVR of the left superior pulmonary vein into the left brachiocephalic vein. A left lower lobe sleeve resection was performed with mediastinal lymph node dissection. Frozen section analysis confirmed the diagnosis of a typical carcinoid tumor with histologically negative bronchial margins. The left superior pulmonary vein PAPVR was then managed by means of administration of systemic heparin, mobilization of the vein, and ligation at its insertion into the brachiocephalic vein. The pericardium was opened, and a vascular clamp was placed across the base of the left atrial appendage. The left superior pulmonary vein was then cut to an appropriate length and anastomosed to the atrial appendage (Figure 2).

After an uneventful recovery, the patient was discharged on postoperative day 4 . The final pathology revealed a T2 N0 M0 typical carcinoid tumor with postobstructive pneumonia of the lower lobe. A follow-up nuclear perfusion study at 3 months demonstrated enhanced flow to the left upper lobe. The patient has done well 30 months after the procedure, with follow-up bronchoscopy demonstrating a well-healed bronchial anastomosis. 